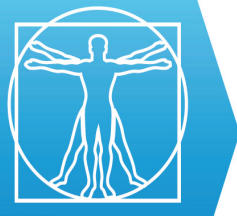

PHYSIOLOGY
1) Department of Physiology, Iuliu Hatieganu University of Medicine and Pharmacy, Cluj-Napoca, Romania

2) Department of Morphopathology, University of Agricultural Sciences and Veterinary Medicine Cluj-Napoca, Romania

3) Department of Surgery, Iuliu Hatieganu University of Medicine and Pharmacy, Cluj-Napoca, Romania

4) Department of Surgical Specialties, Iuliu Hatieganu University of Medicine and Pharmacy, Cluj-Napoca, Romania

\section{Neuronal apoptosis can be prevented by the combined therapy with melatonin and hypothermia in a neonatal rat model of hypoxic-ischemic encephalopathy}

Alina Mihaela Toader ${ }^{1}$, Oana Hoteiuc ${ }^{1}$, Cristina Bidian ${ }^{1}$, Dan-Daniel Oltean ${ }^{4}$, Flaviu Tabaran ${ }^{2}$, Ovidiu Grad $^{3}$, Simona Clichici ${ }^{1}$, Daniela Rodica Mitrea ${ }^{1}$

\begin{abstract}
Introduction. Birth hypoxia is a leading cause of perinatal mortality and neurological morbidity, resulting in central nervous system injury. Cerebral hypoxia and ischemia can produce a severe brain damage following a typical pattern, defined by selective vulnerability of the brain regions. The neonates are most prone to hypoxic-ischemic injuries due to the lack of efficient antioxidant defense. Neonatal hypoxia-ischemia (HI) in a 7-day-old rat $\mathrm{HI}$ model can produce cell death by apoptotic or necrotic mechanisms. The degree of apoptotic or necrotic mechanisms responsible for cell death in neonatal hypoxia-ischemia are not very clear as yet. The form of neuronal death may also depend on the severity of ischemic injury. Necrosis predominates in more severe cases, whereas apoptosis occurs in areas with milder ischemic injury. A human study demonstrated apoptotic and necrotic forms of cell death after hypoxic injury, whereas in some brains from stillbirths, only apoptotic figures were observed. The expression of activated caspase-3 reflects the role of apoptosis in neonatal hypoxic ischemic brain injury.

Objectives. The aim of this study was to evaluate the possible neuroprotective effect of melatonin and hypothermia in hypoxic-ischemic encephalopathy in newborn rats. Local damages induced by hypoxia and ischemia were assessed by evaluating the changes in terms of histology and apoptosis.

Methods. The experiment was conducted on 20 newborn Wistar rats premedicated for seven days with melatonin in a dose of $20 \mathrm{mg} / \mathrm{kg} /$ day. On the $7^{\text {th }}$ postnatal day (P7), the newborn rats were exposed to ischemia (by clamping the right carotid artery) and hypobaric hypoxia ( $8 \% \mathrm{O}_{2}$ for 90 minutes) and some groups to hypothermia.

Results. In this experimental model of neonatal encephalopathy, melatonin, in a dose of $20 \mathrm{mg} / \mathrm{kg} /$ day has neuroprotective effect by reducing the number of cells expressing apoptosis in Cornu Ammonis (CA) (Ammon's Horn) CA1, CA2, CA3 and dentate gyrus of the hippocampus when combined with hypothermia.

Conclusion. The results of this study prove that melatonin is protective in ischemichypoxic brain injuries, but the protection is conditioned in most of the brain regions (excepting cerebral cortex) by conjugation with post-injury hypothermia treatment.

Keywords: neuroprotection, hypoxic-ischemic encephalopathy, apoptosis, melatonin, hypothermia
\end{abstract}

DOI: $10.15386 / \mathrm{mpr}-1903$

Manuscript received: 16.09 .2020

Received in revised form: 10.01.2021

Accepted: 01.02.2021

Address for correspondence:

alina.toader@yahoo.com

This work is licensed under a Creative Commons Attribution-NonCommercialNoDerivatives 4.0 International License

\section{Introduction}

Perinatal hypoxic ischemic brain injury due to acute perinatal asphyxia produces hypoxic-ischemic encephalopathy (HIE) in term and preterm neonates. HIE in newborns is a major cause of death and disability, as up to $60 \%$ of neonates with moderate or severe HIE die during the neonatal period, and at least
$25 \%$ of the survivors have significant longterm neurodevelopmental consequences [1] such as epilepsy, mental retardation, or cerebral palsy [2]. Our focus is the neuroprotection offered by hypothermia in an experimental model of neonatal HIE, also evaluating the combined therapy with melatonin, both having well known cerebral protective effects. 
Brain injury in HIE is a complex evolving process initiated at the time of the primary insult extending into the recovery period. HIE results from two recognized phases: primary and secondary energy failure $[3,4]$. Primary energy failure occurs during the $\mathrm{HI}$ insult and is characterized by decreased cerebral blood flow, which in turn reduces delivery of oxygen and substrates to brain tissue [5]. Following primary energy failure, the cerebral metabolism may shortly recover, only to deteriorate from secondary energy failure, a new phase of neuronal damage, starting at about 6-24 hours after the initial injury mostly defined by mitochondrial dysfunction and initiation of the apoptotic chain reaction. The interval between primary and secondary energy failure represents a latent phase with restoration of cellular energy metabolism, offering an optimal window for neuroprotective interventions such as therapeutic hypothermia. The primacy of therapeutic hypothermia in treating HIE is well established and cooling to $33-34^{\circ} \mathrm{C}$ for $72 \mathrm{~h}$ may be the most appropriate method.

Hypothermia decreases metabolic rate and the cerebral blood flow (CBF) [6], which is altered during different types of injury. Brain oxygenation and glucose concentration decrease during hypoxic and ischemic conditions and cause adenosine triphosphate (ATP) depletion, lactic acid accumulation and acidosis that eventually leads to cell death [7]. Hypothermia reduces cerebral oxygen consumption, it tends to increase hemoglobin oxygen-binding affinity, and inhibits the synthesis, release, and/or reuptake of ischemiainduced neurotransmitters and neuromodulators, including glutamate, glycine, gamma aminobutyric acid (GABA), dopamine, norepinephrine, serotonin, and adenosine $[8,9]$. Mild to moderate hypothermia inhibits the release of reactive oxygen species including hydrogen peroxide [10], malondialdehyde (MDA) [11], and hydroxyl radical formation. Post-hypoxic hypothermia reduces neuronal apoptosis, the production of oxygen free radicals and the amount of excitatory amino acids.

Melatonin (N-acetyl-5-methoxytryptamine) is a natural hormone secreted by the pineal gland and extra pineal tissues and others such as retina, gut, bone marrow, kidney, astrocytes, platelets, and glia cells and has been used in therapies for decades in various diseases due to its antioxidative and antiapoptotic properties [12-14]. Melatonin is an ideal neuroprotective agent as it readily crosses the blood-brain barrier and lacks toxicity in comparison to other neuro-protectants [15]. There is evidence supporting the role of melatonin in regulation of various physiological functions like modulation of sleep, mood, behavior, anti-inflammatory activities, radical scavenging, immunomodulatory activities, antiangiogenic activity, and anti-carcinogenic properties, and so forth [16-20]. Melatonin receptors are widely distributed in central nervous system (CNS), as well as in the peripheral organs which acts as a lipophilic and hydrophilic molecule and able to pass the morpho-physiological barriers such as the blood-brain barrier (BBB) [21-23].

\section{Working hypothesis. Aims}

The enhanced vulnerability of the neonatal brain to hypoxia and ischemia is partially due to the lack of an efficient antioxidant defense, so a pretreatment with an antioxidant may be useful. Regarding neuroprotective strategies, mild hypothermia is a part of the protocol in case of ischemic hypoxic encephalopathy. Our major aim was to test the possible protective effect of a pretreatment with melatonin applied to newborn rats subjected to hypoxia and ischemia, and to evaluate if hypothermia can improve the neuroprotection eventually produced by melatonin.

\section{Materials and methods}

The experimental study was carried out in the research center of the Biobase and Physiology department of the Iuliu Hatieganu University of Medicine and Pharmacy, Cluj-Napoca. The experimental protocol was approved by the Ethics Committee and was carried out in accordance with the legislation.

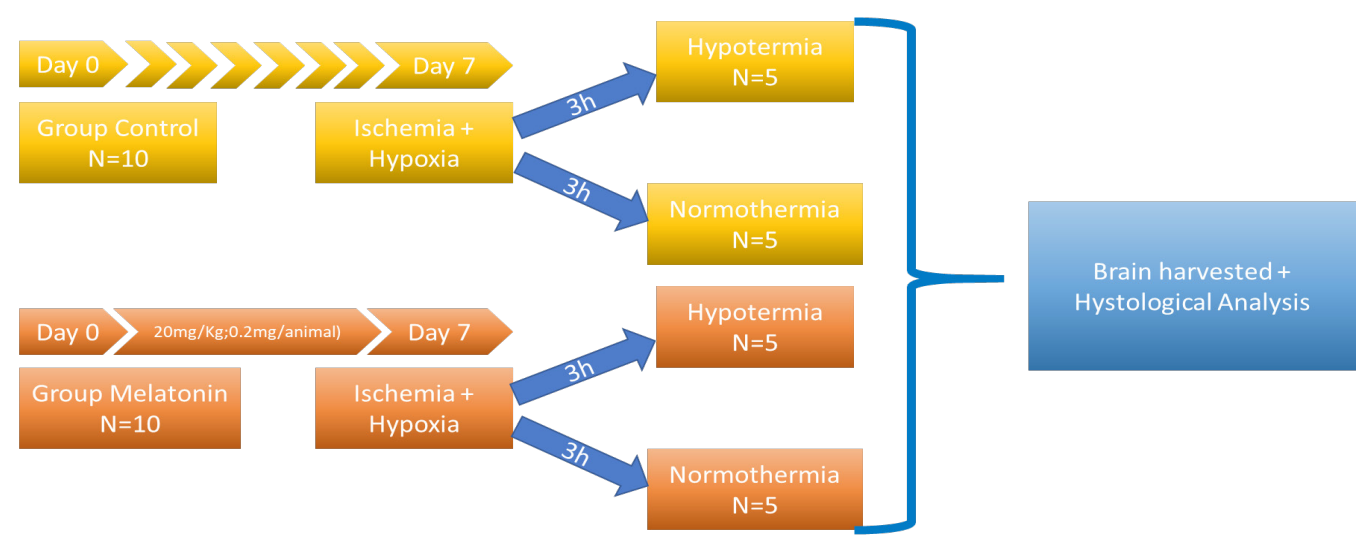

Figure 1. Experiment design diagram. 


\section{Experimental design}

The experiment was conducted on 20 newborn Wistar rats, males and females, divided into groups as shown in figure 1.

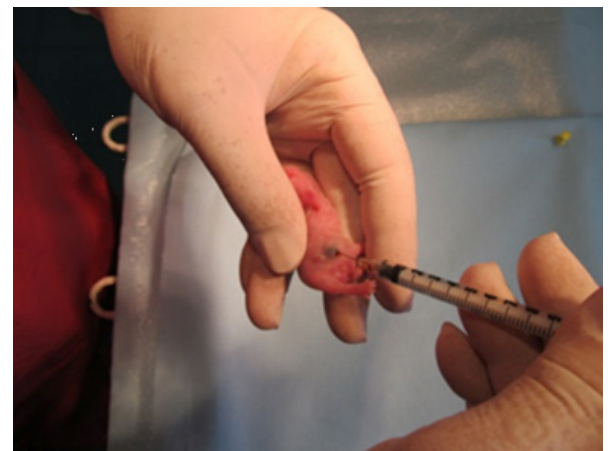

Figure 2. Melatonin injection.
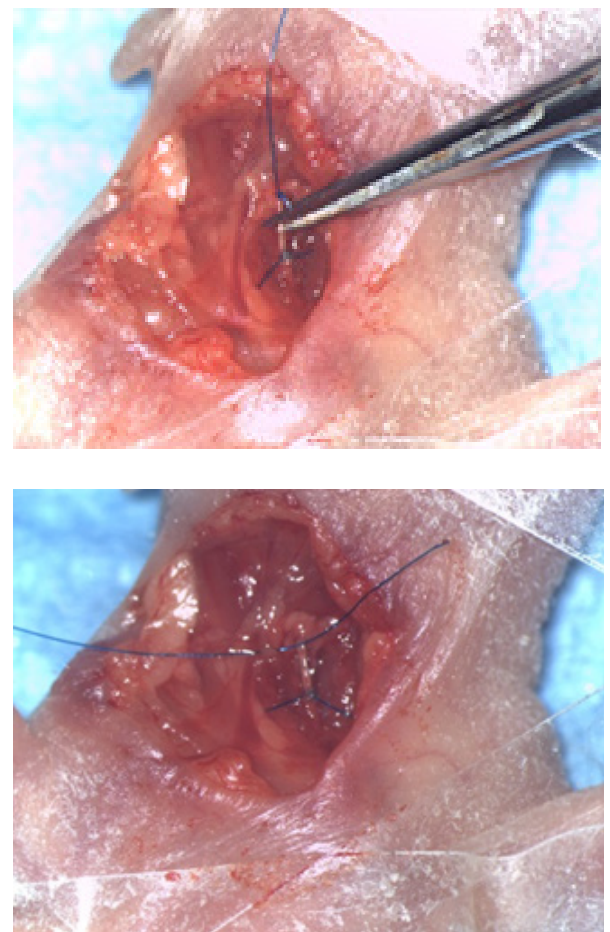

Figure 3 and 4. Carotid artery ligation.

For the pretreated group melatonin was administered in a dose of $20 \mathrm{mg} / \mathrm{kg} /$ day intraperitoneally for seven days as premedication from day 0 (birth). On day 7 the animals were exposed to ischemia (by right carotid artery ligation) and hypobaric hypoxia $\left(8 \% \mathrm{O}_{2}\right.$ for 90 minutes) in barocamera, following a protocol previously described by Levine. The rats were anesthetized by local instillation of xyline. The operation did not exceed $5 \mathrm{~min}$ for each animal. To test the effect of combined therapy of melatonin with hypothermia several animals were exposed after hypoxic-ischemic injury to whole body hypothermia, $33-34^{\circ} \mathrm{C}$ intrarectal, for 3 hours [24]. The animals were euthanized, and the brain was harvested immediately after the end of the experiment.

\section{Histopathological examination}

The harvested tissues were fixed in $10 \%$ paraformaldehyde, $\mathrm{pH} 7$ and processed by the classical paraffin technique. The dehydration was made by successive exposure to ethanol $(70 \%, 95 \%$, and $100 \%)$ for $3 \mathrm{~h}$, then xylene exposure for $45 \mathrm{~min}$ and finally paraffin embedded overnight.

Multiple sections were cut by rotative microtome and mounted onto histological slides. The sections were stained using the Hematoxylin-Eosin technique: the samples were de-paraffined by exposure to xylene, 3 times for $5 \mathrm{~min}$ each, followed by the rehydrating process by immersing the samples into 3 successive solutions of ethylic alcohol $100 \%, 96 \%$ and $70 \%$, for 3 min each, then the slides were washed with water for $5 \mathrm{~min}$, followed by Hematoxylin-Eosin staining.

To process the images, we used an Olympus BX51 microscope, an Olympus SP 350 camera and the Olympus Stream Basic image analysis program. The whole process of harvesting and sectioning of the animal nervous system samples was made according to the INHAND system recommendations.

In brief, the brain regions chosen for examination (Ammon's horn, dentate gyrus, thalamus and cerebral cortex from the parietal and temporal areas) were embedded in paraffin following the routine protocol and finally stained using Hematoxylin eosin technique [24].

The histological sections and the regions chosen for examination are represented in figure 5 .

A

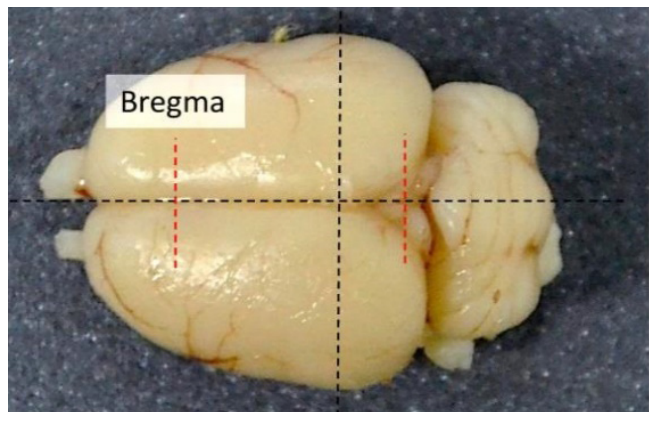

B

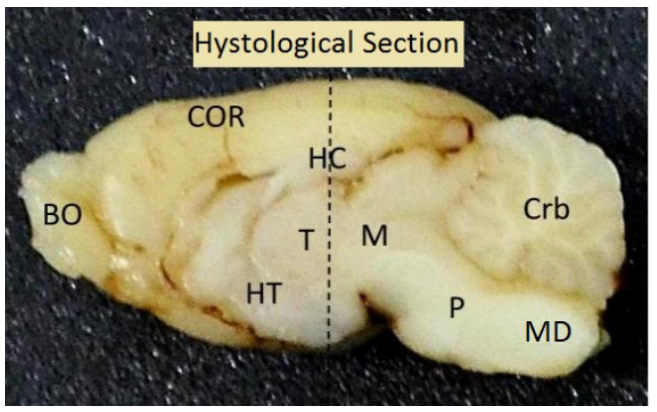

Figure 5. A. The representation of the mark areas and the sectioning plans of the encephalon regions in our experiment. B. The longitudinal section in median plan of the encephalon BO-olfactory bulbs, COR-cerebral cortex, HC-hippocampus, T-thalamus, HT-hypothalamus, M-midbrain, P-pons, Mdmedulla, Crb-cerebellum. 


\section{Immune-histochemistry}

For identification of the neural cells which suffer apoptosis we used the immune-histochemical expression of the Cleaved Caspase 3 (Polyclonal Rabbit antiCaspase3Thermo Scientific-PA1-26426). The immune-histochemical reaction was performed using the Leica Bond-Max TM unit, using a standard indirect avidin-biotin reaction. Quantification of immune-histochemical Cleaved Caspase 3. The expression of Cleaved Caspase 3 was assessed on histological sections from all the 6 regions analyzed: cerebral cortex (COR), thalamus (TA), Ammon's horn (with CA1, CA2, CA3) and dentate gyrus (DG) [24].

\section{Statistical analysis}

For statistical analysis we used the logistic regression method and two-way ANOVA analysis in SPSS Statistics. The results were statistically significant if $\mathrm{p}<0.05$.

\section{Results}

Histological and immuno-histochemical analysis were performed to investigate caspase 3 expression in different brain regions.

In the CA1 hippocampus sub-region, comparing the effects of hypothermia and melatonin, when subjected to hypothermia and hypoxia, the protective effect of the combined therapy with melatonin and hypothermia was noticeable. Hypothermia alone proved to be protective, resulting in the decrease of the number of cas 3 positive cells (Figure 7).

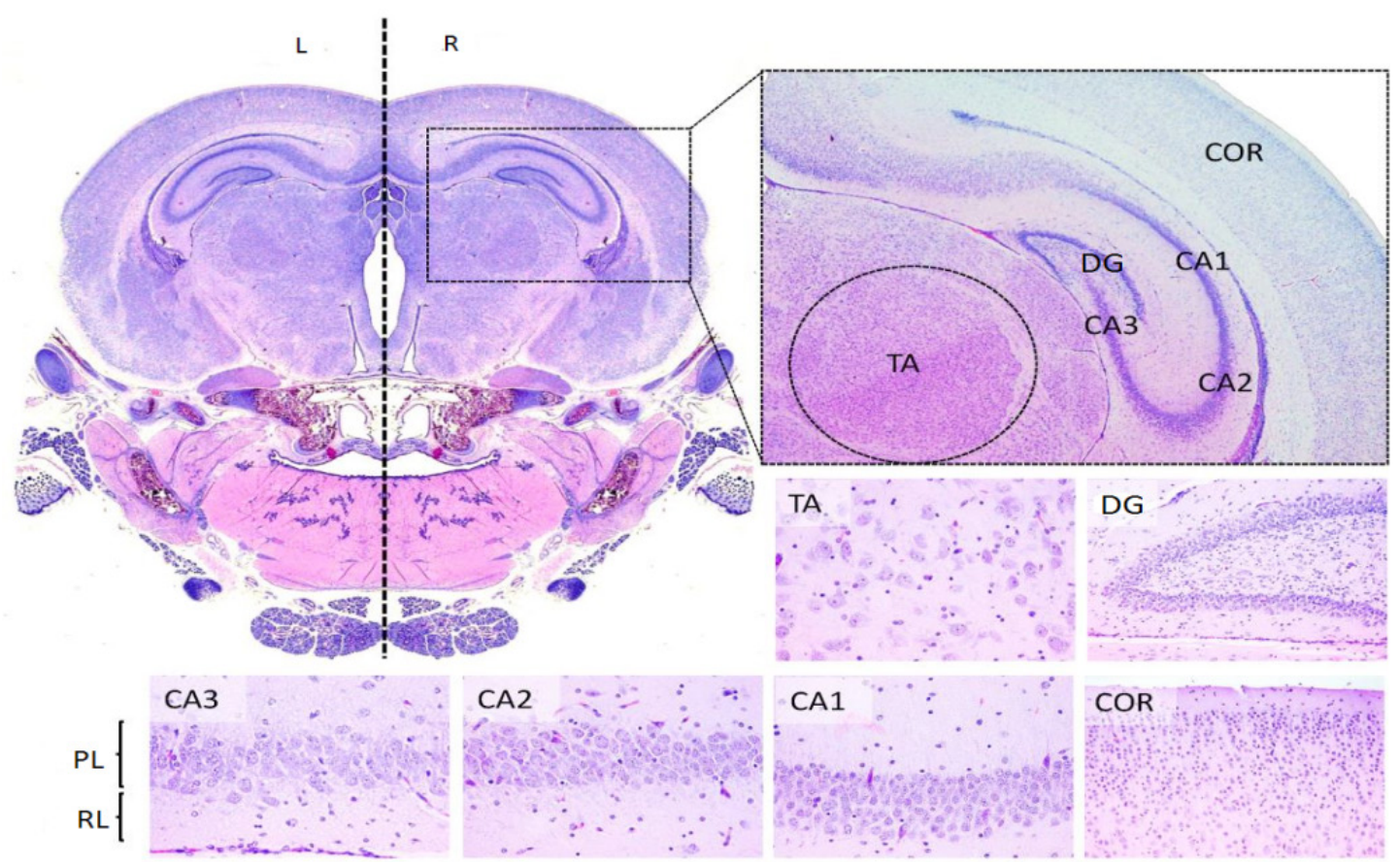

Figure 6. The histological encephalic regions on the $7^{\text {th }}$ postnatal day in rats. The transversal section represents: COR-cortex cerebral, DG-the dentate gyrus, CA- Amon`s Horn (with 3 subregions-CA1, CA2 and CA3), PL- pyramidal cells layer a CA, RL-the radial cells layer of CA, TA-thalamus.

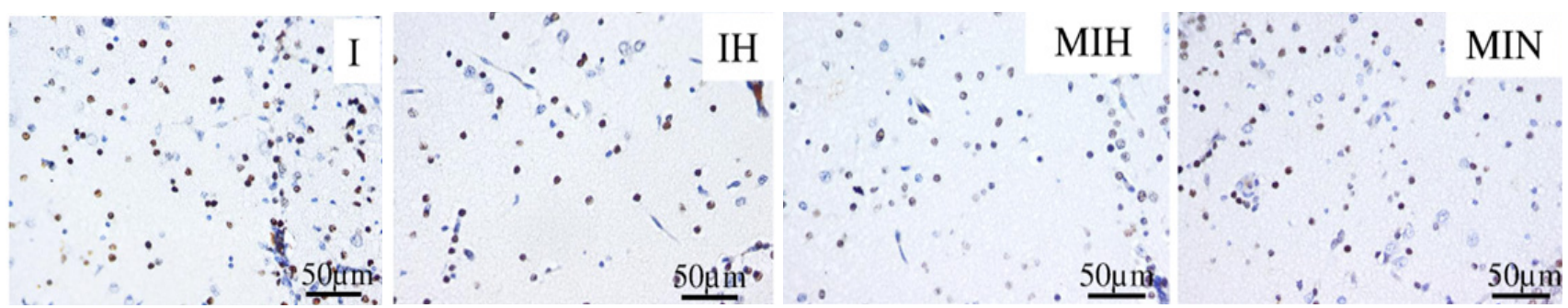

Figure 7. Immune-histochemical expression for activated caspase 3 (cas 3 ) in the radial cells of the Ammon`s horn CA1 sub region. Groups: IH-group exposed to cerebral ischemia and hypothermia IN- group exposed to cerebral ischemia and hypoxia, in normal temperature conditions MIH-group melatonin-ischemia-hypothermia, MIN-group melatonin-ischemia-normal thermic conditions. HE staining, x 40 . 


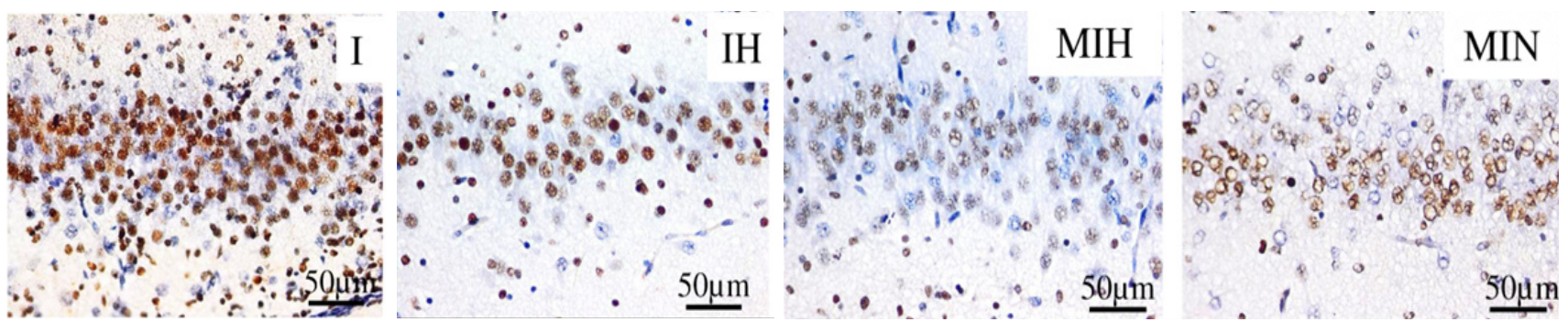

Figure 8. Immune-histochemical expression for activated caspase 3 (cas3) in the pyramidal cells layer of the Ammon`s horn CA3 sub region. Groups: IH-group exposed to cerebral ischemia and hypothermia IN- group exposed to cerebral ischemia and hypoxia, in normal temperature conditions MIH-group melatonin-ischemia-hypothermia, MIN-group melatonin-ischemia-normal thermic conditions. HE staining, $\mathrm{x} 40$.
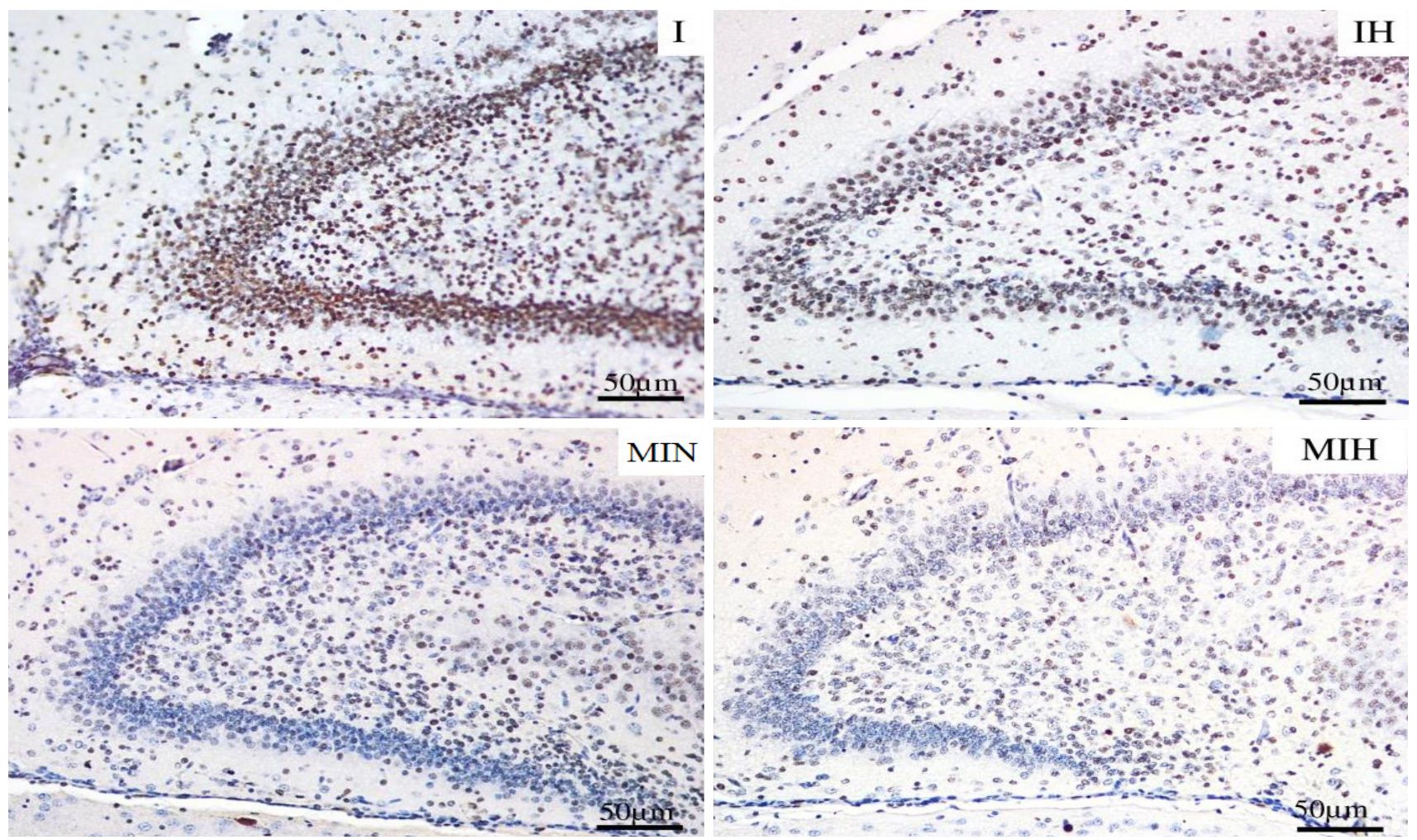

Figure 9. Immune-histochemical expression for activated caspase 3 (cas3) in the dentate gyrus. Groups: IH-group exposed to cerebral ischemia and hypothermia IN- group exposed to cerebral ischemia and hypoxia, in normal temperature conditions MIH-group melatoninischemia-hypothermia, MIN-group melatonin-ischemia-normal thermic conditions. HE staining, x20.
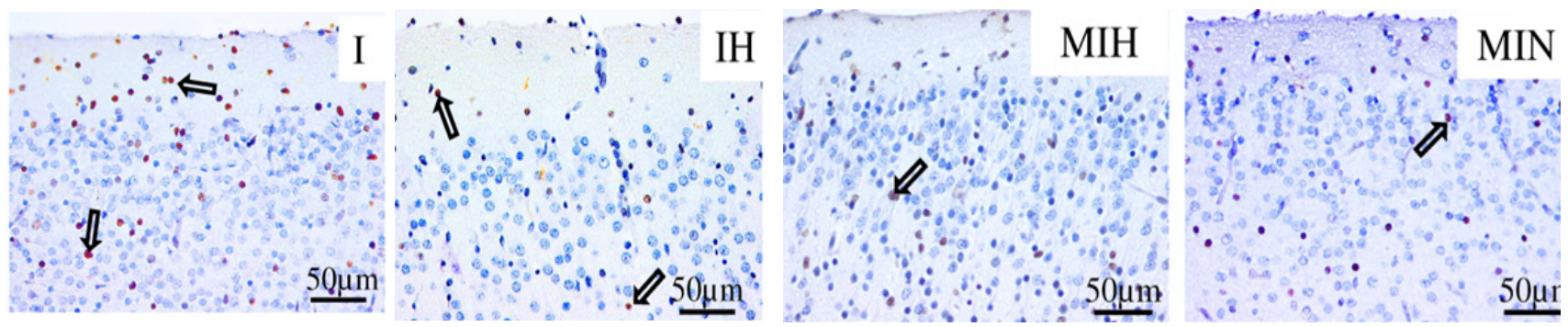

Figure 10. Immune-histochemical expression for activated caspase 3 (cas3) in the cerebral cortex. Groups: IH-group exposed to cerebral ischemia and hypothermia IN- group exposed to cerebral ischemia and hypoxia, in normal temperature conditions MIH-group melatoninischemia-hypothermia, MIN-group melatonin-ischemia-normal thermic conditions. HE staining, $\mathrm{x} 40$. 

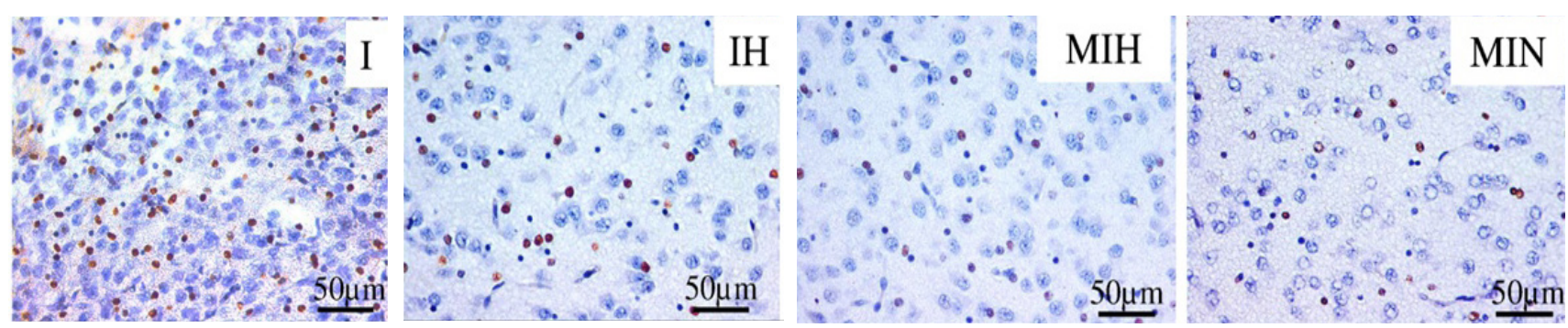

Figure 11. Immune-histochemical expression for activated caspase 3 (cas3) in the thalamus. Groups: IH-group exposed to cerebral ischemia and hypothermia IN-group exposed to cerebral ischemia and hypoxia, in normal temperature conditions MIH-group melatoninischemia-hypothermia, MIN-group melatonin-ischemia-normal thermic conditions. HE staining, x 40.

Regarding the CA3 sub-region, the protective effect appeared in case of using hypothermia as post-treatment. Melatonin was also neuroprotective, the number of cas3 positive cells being reduced (Figure 8).

In the dentate gyrus, the most protective effect was assured by therapeutic hypothermia. Melatonin proved to be protective only when conjugated with hypothermia (Figure 9).

In the cerebral cortex the improvement of the lesions induced by the exposure to cerebral ischemia and hypoxia was observed in melatonin pretreated groups, and the protective effect was not necessarily conditioned by the therapeutic association with hypothermia, but most protection was provided when this combination occurred (Figure 10).

In the thalamic region, melatonin reduced the number of cas3 positive cells, independently of hypothermia being associated as treatment (Figure 11).
The expression of caspase 3 in different brain region was quantified as the percentage of positive stained cells out of the total number of cells.

The protective effect of hypothermia consisting in the reduction of the percentage of cas 3 positive cells was statistically significant $(\mathrm{p}<0.05)$ in the pyramidal layer of the CA1 region $(\mathrm{p}=0.008)$ and $\mathrm{CA} 3$ brain region $(p=0.042)$. Hypothermia reduced the cas 3 expression in all brain regions, but not statistically significantly. See table I for details.

The associated treatment with melatonin produced a significant effect $(p<0.05)$ - reducing cas 3 positive cell percentage when combined with hypothermia, in hippocampus sub-region CA2 $(\mathrm{p}=0.038)$ and CA3 $(p=0.007)$. Hypothermia when combined with melatonin decreased the number of apoptotic cells in all studied regions. See table II for details.

Table I. Cas 3 expression in IH and IN group.

\begin{tabular}{|c|c|c|c|c|c|c|c|c|c|}
\hline \multirow{3}{*}{ Group } & \multicolumn{8}{|c|}{ Brain region (\%) } & \multirow[b]{3}{*}{ Total } \\
\hline & \multicolumn{2}{|c|}{ CA1 } & \multicolumn{2}{|c|}{ CA2 } & & & & & \\
\hline & RL & PL & $\mathbf{R L}$ & $\mathbf{P L}$ & CA3 & Cortex & DG & Thalamus & \\
\hline IN & 49 & 48 & 46.3 & 69 & 90.6 & 17 & 89.6 & 21.6 & 53.8 \\
\hline IH & 40 & 21.6 & 42.6 & 41 & 84 & 13.6 & 84.3 & 16 & 42.8 \\
\hline$p$ (t-test) & 0.416 & 0.008 & 0.429 & 0.202 & 0.042 & 0.473 & 0.315 & 0.272 & 0.041 \\
\hline
\end{tabular}

$\mathrm{IN}=$ Ischemia+hypoxia and Normothermia group; $\mathrm{IH}=$ Ischemia + hypoxia and Hypothermia group.

Table II. Cas3 expression in melatonin treated groups MIH and MIN.

\begin{tabular}{|c|c|c|c|c|c|c|c|c|c|}
\hline \multirow{3}{*}{ Group } & \multicolumn{8}{|c|}{ Brain region (\%) } & \multirow[b]{3}{*}{ Total } \\
\hline & \multicolumn{2}{|c|}{ CA1 } & \multicolumn{2}{|c|}{ CA2 } & \multirow[b]{2}{*}{ CA3 } & \multirow[b]{2}{*}{ Cortex } & \multirow[b]{2}{*}{ DG } & \multirow[b]{2}{*}{ Thalamus } & \\
\hline & $\mathbf{R L}$ & PL & $\mathbf{R L}$ & PL & & & & & \\
\hline МIH & 43.5 & 13.5 & 35 & 55 & 72 & 11.5 & 67.5 & 18.5 & 39.56 \\
\hline MIN & 49.6 & 29.6 & 61 & 58.6 & 91.6 & 12 & 92.6 & 24 & 52.37 \\
\hline$p$ (t-test) & 0.783 & 0.368 & $\mathbf{0 . 0 3 8}$ & 0.835 & 0.007 & 0.952 & 0.285 & 0.390 & 0.139 \\
\hline
\end{tabular}

$\mathrm{MIN}=$ Melatonin and Ischemia+hypoxia, Normothermia group; MIH = Melatonin and Ischemia + hypoxia, Hypothermia group) . 
Table III. Cas3 expression in hypothermic groups IH and MIH.

\begin{tabular}{|c|c|c|c|c|c|c|c|c|c|}
\hline \multirow{3}{*}{ Group } & \multicolumn{8}{|c|}{ Brain region (\%) } & \multirow[b]{3}{*}{ Total } \\
\hline & \multicolumn{2}{|c|}{ CA1 } & \multicolumn{2}{|c|}{ CA2 } & & & & & \\
\hline & $\mathbf{R L}$ & $\mathbf{P L}$ & $\mathbf{R L}$ & $\mathbf{P L}$ & CA3 & Cortex & DG & Thalamus & \\
\hline IH & 49 & 48 & 46.3 & 69 & 90.6 & 17 & 89.6 & 21.6 & 53.8 \\
\hline МIH & 43.5 & 13.5 & 35 & 55 & 72 & 11.5 & 67.5 & 18.5 & 39.56 \\
\hline$p$ (t-test) & 0.717 & 0.017 & 0.095 & 0.460 & 0.002 & 0.422 & 0.324 & 0.373 & 0.004 \\
\hline
\end{tabular}

IN = Ischemia +hypoxia and Normothermia group; IH = Ischemia+hypoxia and Hypothermia group.

Table IV. Cas3 expression in normal thermic condition groups IN and MIN.

\begin{tabular}{|c|c|c|c|c|c|c|c|c|c|}
\hline \multirow{3}{*}{ Group } & \multicolumn{8}{|c|}{ Brain region (\%) } & \multirow[b]{3}{*}{ Total } \\
\hline & \multicolumn{2}{|c|}{ CA1 } & \multicolumn{2}{|c|}{ CA2 } & \multirow[b]{2}{*}{ CA3 } & \multirow[b]{2}{*}{ Cortex } & \multirow[b]{2}{*}{ DG } & \multirow[b]{2}{*}{ Thalamus } & \\
\hline & $\mathbf{R L}$ & $\overline{\mathbf{P L}}$ & $\mathbf{R L}$ & $\overline{\mathbf{P L}}$ & & & & & \\
\hline MIN & 40 & 21.6 & 42.6 & 41 & 84 & 13.6 & 84.3 & 16 & 42.8 \\
\hline IN & 49.6 & 29.6 & 61 & 58.6 & 91.6 & 12 & 92.6 & 24 & 52.37 \\
\hline$p$ (t-test) & 0.564 & 0.534 & 0.100 & 0.385 & 0.058 & 0.677 & 0.170 & 0.232 & 0.196 \\
\hline
\end{tabular}

$\mathrm{MIN}=$ Melatonin and Ischemia+hypoxia, Normothermia group; MIH = Melatonin and Ischemia+hypoxia, Hypothermia group).

Melatonin pretreatment when combined with hypothermia reduced statistically significantly $(p=0.017)$ the number of cas 3 positive cells in the hippocampus subregion CA1 $(p=0.017)$ and CA3 $(p=0.002)$. In all brain regions studied, the percentage of cas 3 positive cells was significantly reduced in the melatonin pretreated group $(\mathrm{p}=0.004)$. See table III for details.

In normal thermic conditions there were no statistically significant differences in the studied groups. Melatonin pretreatment shrank the number of cas 3 positive cells, not statistically significantly $(p>0.05)$. See table IV for details.

\section{Multivariate analysis}

The two-way Anova analysis showed that in Radial Layer (RL) CA1, PL CA2, cortex and thalamus melatonin pretreatment and hypothermia did not produce significant effects on the cas 3 expression.

In the other regions the effects of the melatonin pretreatment and hypothermia were significant. In the Pyramidal Layer (PL) of the CA1 sub-region we found a combined significant effect on the cas 3 expression $(\mathrm{p}=0.024)$, as shown in figure 12 .

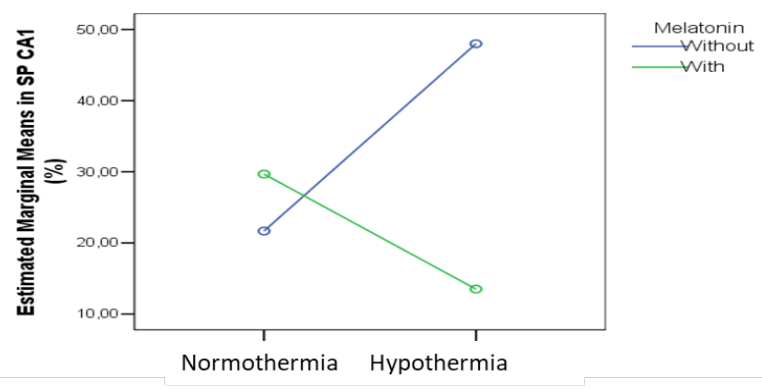

Figure 12. Cas 3 expression means in \% in PL of CA1 in groups $\mathrm{IH}, \mathrm{IN}, \mathrm{MIH}$ and MIN.
In the RL CA2 we also found a significant combined effect on the cas 3 expression $(\mathrm{p}=0.021)$ (Figure 13).

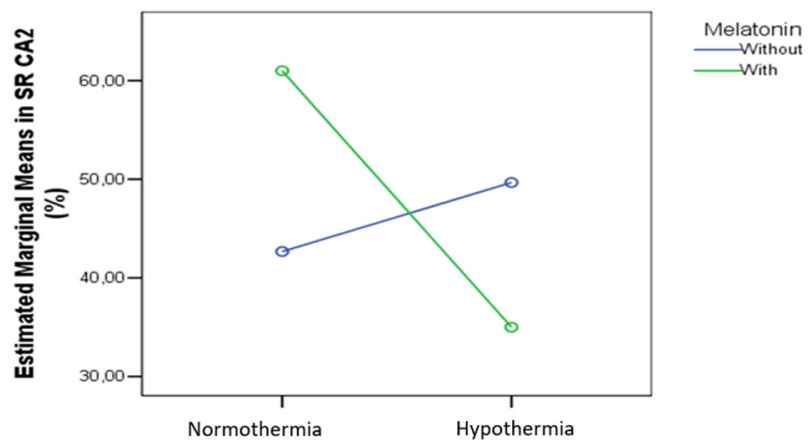

Figure 13. Cas 3 expression means in $\%$ in $\mathrm{RL}$ of the $\mathrm{CA} 2$ region in groups IH, IN, MIH and MIN.

In the $\mathrm{CA} 3$ region we found a significant melatonin $(\mathrm{p}=0.02)$ and hypothermia $(\mathrm{p}=0.01)$ effect $(\mathrm{p}=0.02)$ and a combined effect statistically significant $(\mathrm{p}<0.001)$ on the cas3 expression, as in figure 14.
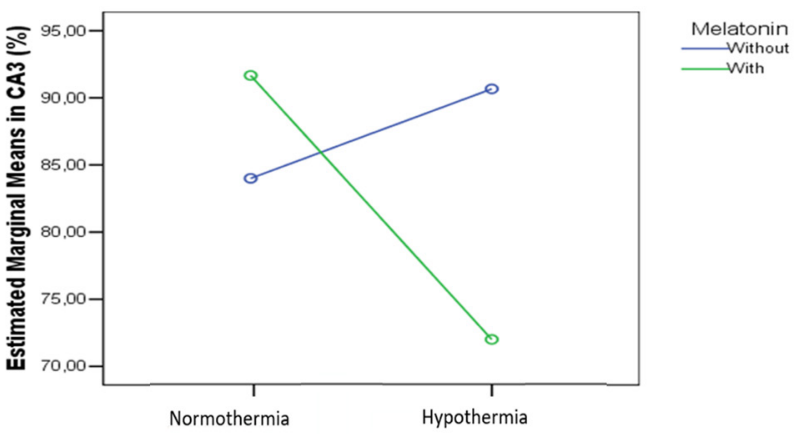

Figure 14. Cas 3 expression means in \% in CA3 region. 
In the DG we found a significant combined effect on the cas 3 expression ( $\mathrm{p}=0.019)$, as per figure 15 .

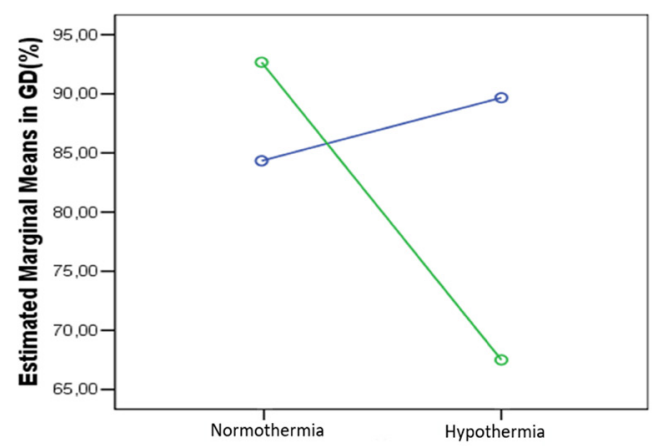

Figure 15. Cas 3 expression means in \% in DG region.

\section{Discussion}

The most affected areas of newborn rats during unilateral ischemia-hypoxia, in order of severity of lesions, were the dentate gyrus and the sub-region CA3 of the hippocampus. The CA1, the thalamus and cerebral cortex were less affected. In the CA3 sub-region of the hippocampus, comparing the effects of melatonin and low temperature we observed the protective effect of the combination of melatonin and therapeutic hypothermia (33$34^{\circ} \mathrm{C}$ rectal temperature). As with CA1 sub-region we have found that melatonin combined with hypothermia therapy does not provide superior protection to hypothermia alone. The distribution of the lesions in the cerebral cortex is specific, the cells which undergo apoptosis being arranged mainly in the layer I of the cortex. This is because neuronal population sensitivity in the brain exposed to ischemia and hypoxia is variable and dependent on the duration and severity of the lesion process and is quantified by the expression of the activated caspase3. Ammon's horn neurons in area CA1 are most sensitive to depolarization due to ischemia (3-5 minutes) compared to neurons located in the striated layer, neurons that resists in average between 15 and 20 minutes [20]. Regarding the type of neuronal cell death, necrotic lesions predominate in high intensity ischemia, whereas apoptosis occurs when ischemic damage is mild or moderate. Also, the apoptotic lesions have a much slower progress compared with the necrotic one. Other studies also demonstrate the morphological and functional benefits of early initiation of hypothermia in conjunction with melatonin in unilateral brain ischemia and hypoxia $[21,22]$. Thus, hypothermia provides neuroprotection, clinically improving the behavioral performance in both adults and neonates. Studies in adults prove that hypothermia initiated immediately after cardiac arrest provides significant improvements and the late cooling after brain trauma appears to be effective. Children born at term suffering from hypoxic-ischemic encephalopathy and who were less severely affected, were exposed for 72 hours to moderate systemic hypothermia (rectal temperature of $34.5^{\circ} \mathrm{C}$ ), and the results were promising with reduced neuronal death and disability $[22,24]$.

Melatonin activity is mediated by the specific receptors in cellular membranes by two high affinity melatonin receptors, melatonin receptor (MT) MT1 and MT2 [23,25]. Amphiphilic melatonin being a potent antioxidative and antiapoptotic agent has been used clinically in various CNS disorders [26]. It has been used in various neurodegenerative diseases like Alzheimer's, Parkinson, and stroke due to its property of inhibiting apoptotic pathways and by activating survival pathways due to its protective properties [27]. Melatonin has conferred a cerebral-protective effect, as shown by reduced infarct volume, lowered brain edema, and increased neurological scores [28]. Melatonin $(5 \mathrm{mg} / \mathrm{kg})$ pretreatment intraperitoneally has diminished reactive oxygen species (ROS) levels, and inhibited cell apoptosis which may contribute to its antioxidant and antiapoptotic effects during brain ischemia [29]. Melatonin and its metabolites have been able to modulate the oxidative stress by reducing ROS, MDA, and NO and restored the GSH and SOD level in various ischemic studies [32,33].

It has been shown that melatonin is an effective antioxidant in various in vitro and in vivo models of neurodegenerative diseases not only by scavenging free radicals but also by increasing the gene expression of antioxidative enzymes like glutathione peroxidase (GPx), glutathione reductase (GR), and superoxide dismutase (SOD) [34]. It has also decreased the expression of apoptotic factor Bax [29-37].

Melatonin acts via high affinity G-protein-coupled melatonin receptors, MT1 and MT2. The specific receptors have been found in human brain regions like medial preoptic area, anterior hypothalamus, paraventricular and anteroventral thalamic nuclei, hippocampus, cerebral and cerebellar cortex, and retina [23].

Melatonin has been found effective in various animal models of brain injury. Several research studies are being done on assessing protective role of melatonin in humans. Melatonin, a potent antiapoptotic and antioxidative neuroprotectant with no serious toxicity, raises hopes that it might be used for neonates for HIE treatment. In our study melatonin turned out to be neuroprotectant, especially when combined with hypothermia, in all cerebral regions elected for the study.

Regarding the remarkable effects of hypothermia, even a small reduction in temperature provides significant neuroprotection after ischemic neuronal injury [38]. In addition to modulating the numerous damaging processes initiated by ischemia, hypothermia also affects metabolic rate and the cerebral blood flow $(\mathrm{CBF})[6]$, which is altered during cerebral hypoxia and ischemia. CBF is usually reduced after focal or global ischemia. Some studies show 
that hypothermia increases CBF during ischemia [39], while others suggest that it has no effect [40]. Brain oxygenation and glucose concentration decrease during hypoxic and ischemic conditions and cause ATP depletion, lactic acid accumulation and acidosis that eventually leads to cell death [7]. Hypothermia reduces cerebral oxygen consumption, tends to increase hemoglobin oxygenbinding affinity, and inhibits the synthesis, release, and/ or reuptake of ischemia-induced neurotransmitters and neuromodulators, including glutamate, glycine, GABA, dopamine, norepinephrine, serotonin, and adenosine $[8,9]$. Mild to moderate hypothermia inhibits the release of reactive oxygen species including hydrogen peroxide [10], thiobarbituric acid-reactive substances produced after hemorrhage shock [11], and hydroxyl radical formation [41].

Primary energy failure is associated with acute intracellular derangements, including loss of membrane ionic homeostasis, release and blocked reuptake of excitatory neurotransmitters, defective osmoregulation, and inhibition of protein synthesis [42]. Stimulation of excitatory neurotransmitter receptors causes an increase in intracellular calcium, which in turn sets off a cascade of destructive pathways, ultimately resulting in acute cell death and necrosis [43]. The pathogenesis of secondary energy failure is not as well understood as primary energy failure, but likely involves multiple pathophysiologic processes, including the accumulation of excitatory neurotransmitters such as glutamate, oxidative injury, anaerobic metabolism and free radical production, inflammation, and ultimately initiation of apoptosis. The secondary phase of injury occurs slowly (within hours to days) in the setting of normal intracellular $\mathrm{pH}$ and stable cardiorespiratory status [3]. Much of the injury following secondary energy failure is related to apoptosis. Although acute cellular necrosis and apoptosis are both noted in post-hypoxic-ischemic injury in animal models and human infants, apoptotic cell death appears to be a more significant contributor to damage in the developing brain and is the major component of secondary energy failure $[44,45]$.

Various studies have examined the beneficial effects of hypothermia at the molecular level in different animal models. It has been determined that post-insult mild therapeutic hypothermia $\left(34.9^{\circ} \mathrm{C}\right.$ for 12 hours) reduces apoptotic cell death in piglets [44]. The effects of prolonged hypothermia $\left(30^{\circ} \mathrm{C}\right.$ for 72 hours) begun immediately after $\mathrm{HI}$ insult have been evaluated in P7 Wistar rats [46]. In this setting, the number of apoptotic cells in the parietal cortex and hippocampus was reduced [46]. Caspase-3 levels in the induced hypothermia groups were lower than in the normothermic group 24-48 hours post HI [45].

The results of our study show the same anti apoptotic effect of mild hypothermia $\left(34^{\circ} \mathrm{C}\right.$, for 3 hours), especially in the cerebral regions most prone to hypoxic-ischemic lesions. Another study of systemic induced hypothermia $\left(30^{\circ} \mathrm{C}\right.$ for 10 hours immediately after $\mathrm{HI}$ insult) in $\mathrm{P} 7$ Wistar rat pups also showed anti-apoptotic neuroprotective effects [47]. The brain infarct volumes as evaluated by loss of MAP-2 staining were reduced in cortex, thalamus, and hippocampus in the hypothermia group animals. The activation of caspase-3 was also significantly reduced at 24 hours post HI injury in the hypothermic group [47]. These results indicate that hypothermia may act at least partially through inhibition of the intrinsic pathway of caspase activation in the neonatal brain, thereby preventing apoptotic cell death. Mild hypothermia $\left(34^{\circ} \mathrm{C}\right)$ decreases expression of caspase- 3 mRNA and lowers caspase- 3 enzyme activity [48]. In infants with HIE, hypothermia was induced within 6 hours after the insult and when continued until 24 hours or longer, improved cell viability at 48 hours [49].

The evidence strongly suggests that hypothermia can effectively improve outcomes of neonate HIE [50,51]. To date, hypothermia has been incorporated into clinical practice as the standard of care for infants with HIE. Although hypothermia has been a revolutionary therapy in HIE, clinical trials have shown that more than $40 \%$ of the cooled infants either died or survived with impairments. Therefore, the search for more effective therapeutic interventions in addition to hypothermia continues. This was the idea that started our study. We wanted to find some new associations between two strategies already known to be neuroprotective, and to see if a combined therapy will be beneficial.

Despite the established role of hypothermia as a neuroprotective strategy for HIE, there are gaps in our knowledge. Our study applied therapeutic mild hypothermia for a short period, only for 3 hours, but the optimal duration needs to be clarified further.

\section{Conclusions}

The results of this experiment prove that melatonin can be neuroprotective in hypoxic-ischemic brain injuries, but the protection is conditioned in most of the brain regions (excepting cerebral cortex and thalamus) by conjugation with post-injury mild hypothermia treatment. Our study also showed that hypothermia remains the most effective neuroprotective therapy when applied in hypoxic-ischemic encephalopathy.

\section{References}

1. Vannucci RC. Experimental biology of cerebral hypoxiaischemia: relation to perinatal brain damage. Pediatr Res. 1990;27(4 Pt 1):317-326.

2. Dilenge ME, Majnemer A, Shevell MI. Long-term developmental outcome of asphyxiated term neonates. J Child Neurol. 2001;16:781-792.

3. Lorek A, Takei Y, Cady EB, Wyatt JS, Penrice J, Edwards $\mathrm{AD}$, et al. Delayed ("secondary") cerebral energy failure 
after acute hypoxia-ischemia in the newborn piglet: continuous 48-hour studies by phosphorus magnetic resonance spectroscopy. Pediatr Res. 1994;36:699-706.

4. Perlman JM. Intervention strategies for neonatal hypoxicischemic cerebral injury. Clin Ther. 2006;28:1353-1365.

5. Laptook AR, Corbett RJ, Ruley J, Olivares E. Blood flow and metabolism during and after repeated partial brain ischemia in neonatal piglets. Stroke. 1992;23:380-387.

6. Sahuquillo J, Vilalta A. Cooling the injured brain: how does moderate hypothermia influence the pathophysiology of traumatic brain injury. Curr Pharm Des. 2007;13:23102322.

7. Erecinska M, Thoresen M, Silver IA. Effects of hypothermia on energy metabolism in Mammalian central nervous system. J Cereb Blood Flow Metab. 2003;23:513-530.

8. Globus MY, Busto R, Dietrich WD, Martinez E, Valdes I, Ginsberg MD. Effect of ischemia on the in vivo release of striatal dopamine, glutamate, and gamma-aminobutyric acid studied by intracerebral microdialysis. J Neurochem. 1988;51:1455-1464.

9. Okuda C, Saito A, Miyazaki M, Kuriyama K. Alteration of the turnover of dopamine and 5-hydroxytryptamine in rat brain associated with hypothermia. Pharmacol Biochem Behav. 1986;24:79-83.

10. Lei B, Adachi N, Arai T. The effect of hypothermia on $\mathrm{H} 2 \mathrm{O} 2$ production during ischemia and reperfusion: a microdialysis study in the gerbil hippocampus. Neurosci Lett. 1997;222:91-94.

11. Guven H, Amanvermez R, Malazgirt Z, Kaya E, Doganay $\mathrm{Z}$, Celik C, et al. Moderate hypothermia prevents brain stem oxidative stress injury after hemorrhagic shock. J Trauma. 2002;53:66-72.

12. Zhang HM, Zhang Y. Melatonin: a well-documented antioxidant with conditional pro-oxidant actions. J Pineal Res. 2014;57:131-146.

13. Galano A, Tan DX, Reiter RJ. Melatonin as a natural ally against oxidative stress: a physicochemical examination. J Pineal Res. 2011;51:1-16.

14. García JJ, López-Pingarrón L, Almeida-Souza P, Tres A, Escudero P, García-Gil FA, et al. Protective effects of melatonin in reducing oxidative stress and in preserving the fluidity of biological membranes: a review. J Pineal Res. 2014;56:225-237.

15. Miller E, Morel A, Saso L, Saluk J. Melatonin redox activity. Its potential clinical applications in neurodegenerative disorders. Curr Top Med Chem. 2015;15:163-169.

16. Claustrat B, Leston J. Melatonin: Physiological effects in humans. Neurochirurgie. 2015;61:77-84.

17. Hardeland R, Poeggeler B. Melatonin and synthetic melatonergic agonists: actions and metabolism in the central nervous system. Cent Nerv Syst Agents Med Chem. 2012;12:189-216.

18. Lucke-Wold BP, Smith KE, Nguyen L, Turner RC, Logsdon AF, Jackson GJ, et al. Sleep disruption and the sequelae associated with traumatic brain injury. Neurosci Biobehav Rev. 2015;55:68-77.

19. Jardim BV, Ferreira LC, Borin TF, Moschetta MG, Gelaleti
GB, Lopes JR, et al. Evaluation of the anti-angiogenic action of melatonin in breast cancer. BMC Proc. 2013;7(Suppl 2):P11.

20. Medvedeva YV, Ji SG, Yin HZ, Weiss JH. Differential Vulnerability of CA1 versus CA3 Pyramidal Neurons After Ischemia: Possible Relationship to Sources of $\mathrm{Zn} 2+$ Accumulation and Its Entry into and Prolonged Effects on Mitochondria. J Neurosci. 2017;37:726-737.

21. Korkmaz A, Topal T, Tan DX, Reiter RJ. Role of melatonin in metabolic regulation. Rev Endocr Metab Disord. 2009;10:261-270.

22. Alghamdi BS. The neuroprotective role of melatonin in neurological disorders. J Neurosci Res. 2018;96:1136-1149.

23. Lacoste B, Angeloni D, Dominguez-Lopez S, Calderoni S, Mauro A, Fraschini F, et al. Anatomical and cellular localization of melatonin MT1 and MT2 receptors in the adult rat brain. J Pineal Res. 2015;58:397-417.

24. Toader AM, Cătoi FA, Dogaru G, Tăbăran F, Anescu C, Fărcaş L, et al. The Combined Therapy with Resveratrol and Hypothermia Prevents Apoptosis in a Neonatal Rat Model of Global Hypoxic-Ischemic Encephalopathy, Bulletin USAMV, Veterinary Medicine, 2013;70:173-176.

25. Rios ER, Venâncio ET, Rocha NF, Woods DJ, Vasconcelos S, Macedo D, et al. Melatonin: pharmacological aspects and clinical trends. Int J Neurosci. 2010;120:583-590.

26. Escribano BM, Colin-González AL, Santamaria A, Túnez I. The role of melatonin in multiple sclerosis, Huntington's disease and cerebral ischemia. CNS Neurol Disord Drug Targets. 2014;13:1096-1119.

27. Sarlak G, Jenwitheesuk A, Chetsawang B, Govitrapong P. Effects of melatonin on nervous system aging: neurogenesis and neurodegeneration. J Pharmacol Sci. 2013;123:9-24.

28. Yang Y, Jiang S, Dong Y, Fan C, Zhao L, Yang X, et al. Melatonin prevents cell death and mitochondrial dysfunction via a SIRT1-dependent mechanism during ischemic-stroke in mice. J Pineal Res. 2015;58:61-70.

29. Li H, Wang Y, Feng D, Liu Y, Xu M, Gao A, et al. Alterations in the time course of expression of the Nox family in the brain in a rat experimental cerebral ischemia and reperfusion model: effects of melatonin. J Pineal Res. 2014;57:110-119.

30. Wang X, Figueroa BE, Stavrovskaya IG, Zhang Y, Sirianni $\mathrm{AC}, \mathrm{Zhu} \mathrm{S}$, et al. Methazolamide and melatonin inhibit mitochondrial cytochrome $\mathrm{C}$ release and are neuroprotective in experimental models of ischemic injury. Stroke. 2009;40:1877-1885.

31. Corrales A, Vidal R, Garcia S, Vidal V, Martinez P, Garcia E, et al. Chronic melatonin treatment rescues electrophysiological and neuromorphological deficits in a mouse model of Down syndrome. J Pineal Res. 2014;56:51-61.

32. Ozacmak VH, Barut F, Ozacmak HS. Melatonin provides neuroprotection by reducing oxidative stress and HSP70 expression during chronic cerebral hypoperfusion in ovariectomized rats. J Pineal Res. 2009;47:156-163.

33. Pandi-Perumal SR, BaHammam AS, Brown GM, Spence DW, Bharti VK, Kaur C, et al. Melatonin antioxidative defense: therapeutical implications for aging and neurodegenerative processes. Neurotox Res. 2013;23:267-300. 
34. Wang $X$. The antiapoptotic activity of melatonin in neurodegenerative diseases. CNS Neurosci Ther. 2009; 15:345-357.

35. Chern CM, Liao JF, Wang YH, Shen YC. Melatonin ameliorates neural function by promoting endogenous neurogenesis through the MT2 melatonin receptor in ischemic-stroke mice. Free Radic Biol Med. 2012;52:1634-1647.

36. Rao VK, Carlson EA, Yan SS. Mitochondrial permeability transition pore is a potential drug target for neurodegeneration. Biochim Biophys Acta. 2014;1842:1267-1272.

37. Sims NR, Muyderman H. Mitochondria, oxidative metabolism and cell death in stroke. Biochim Biophys Acta. 2010;1802:80-91.

38. Ginsberg MD, Sternau LL, Globus MY, Dietrich WD, Busto R. Therapeutic modulation of brain temperature: relevance to ischemic brain injury. Cerebrovasc Brain Metab Rev. 1992;4:189-225.

39. Jenkins LW, DeWitt DS, Johnston WE, Davis KL, Prough DS. Intraischemic mild hypothermia increases hippocampal CA1 blood flow during forebrain ischemia. Brain Res. 2001;890:1-10.

40. Sick TJ, Tang R, Perez-Pinzón MA. Cerebral blood flow does not mediate the effect of brain temperature on recovery of extracellular potassium ion activity after transient focal ischemia in the rat. Brain Res. 1999;821:400-406.

41. Globus MY, Alonso O, Dietrich WD, Busto R, Ginsberg MD. Glutamate release and free radical production following brain injury: effects of posttraumatic hypothermia. J Neurochem. 1995;65:1704-1711.

42. Johnston MV, Trescher WH, Ishida A, Nakajima W. Neurobiology of hypoxic-ischemic injury in the developing brain. Pediatr Res. 2001;49:735-741.

43. Jacobson MD. Programmed cell death: a missing link is found. Trends Cell Biol. 1997;7:467-469.

44. Edwards AD, Yue X, Squier MV, Thoresen M, Cady EB, Penrice J, et al. Specific inhibition of apoptosis after cerebral hypoxia-ischaemia by moderate post-insult hypothermia. Biochem Biophys Res Commun. 1995;217:1193-1199.

45. Rothstein RP, Levison SW. Gray matter oligodendrocyte progenitors and neurons die caspase- 3 mediated deaths subsequent to mild perinatal hypoxic/ischemic insults. Dev Neurosci. 2005;27:149-159.

46. Ohmura A, Nakajima W, Ishida A, Yasuoka N, Kawamura M, Miura S, et al. Prolonged hypothermia protects neonatal rat brain against hypoxic-ischemia by reducing both apoptosis and necrosis. Brain Dev. 2005;27:517-526.

47. Zhu C, Wang X, Cheng X, Qiu L, Xu F, Simbruner G, et al. Post-ischemic hypothermia-induced tissue protection and diminished apoptosis after neonatal cerebral hypoxiaischemia. Brain Res. 2004;996:67-75.

48. Wang LS, Yu LJ, Shao XM. Mild hypothermia attenuates neuronal apoptosis after cerebral hypoxia-ischemia in neonatal rats. Zhongguo Dang Dai Er Ke Za Zhi, 2007;9:3741.

49. Hiroi M, Ogihara T, Hirano K, Hasegawa M, Morinobu T, Tamai H, et al. Regulation of apoptosis by glutathione redox state in PC12 cells exposed simultaneously to iron and ascorbic acid. Free Radic Biol Med. 2005;38:1057-1072.

50. Gluckman PD, Wyatt JS, Azzopardi D, Ballard R, Edwards $\mathrm{AD}$, Ferriero DM, Polin RA, Robertson CM, Thoresen M, Whitelaw A, Gunn AJ. Selective head cooling with mild systemic hypothermia after neonatal encephalopathy: multicentre randomised trial. Lancet. 2005,365:663-670.

51. Shankaran S, Laptook AR, Ehrenkranz RA, Tyson JE, McDonald SA, Donovan EF, et al. Whole-body hypothermia for neonates with hypoxic-ischemic encephalopathy. N Engl J Med. 2005,353:1574-1584. 